\title{
Complete Remission of Choriocarcinoma with Primary Mtx Therapy and its Prevention using Mtx
}

\section{Kazuo Maeda*}

Department of Obstetrics and Gynecology (Emeritus), Tottori University Medical School, Yonago, Japan

\begin{abstract}
Aims: There were frequent hematogenous metastases ending with death due to brain metastasis in gestational choriocarcinoma (Ch-Ca) before methotrexate (MTX) therapy around 1960. As the gestational Ch-Ca was a systemic disease, it should be treated by systemic primary chemotherapy before local treatment.

Methods: MTX was administered associating Actinomycin D, with intermittent or continuous regimen until the disappearance of $\mathrm{Ch}-\mathrm{Ca}$ tumor and human chorionic gonadotropin (hCG).

Results: Metastases, primary uterine focus, and hCG disappeared by the chemotherapy. Reproductive function recovered achieving normal uterine pregnancy after complete remission. Ch-Ca brain metastasis was treated by the MTX chemotherapy without recurrence for 20 years.

Prevention of Ch-Ca: MTX was administered to 107 women after the complete hydatidiform mole until negative pregnancy test, where no Ch-Ca developed, while 6 Ch-Ca developed in 81 control cases of no MTX in 1968. An UICC RCT result was the same as above. However, 13 Ch-Cas were reported in 22 Japanese districts 2013, maybe no molar cyst was detected with B-mode image in early pregnancy, followed by no chemotherapy, but would be terminated as blighted ovum. The author calculated the number of estimated $\mathrm{Ch}-\mathrm{Ca}$ cases to be 13 , which was the same as actually reported number of Ch-Ca cases in 2013 and 2014.
\end{abstract}

Keywords: Choriocarcinoma; Primary chemotherapy; MTX; Complete remission; HCG; Hydatidiform mole; Choriocarcinoma prevention

\section{Introduction}

Gestational choriocarcinoma is the cancer of trophpblast, which is the epithelium covering placental villi, namely it is fetal origin, and develops mainly after complete hydatidiform mole pregnancy. Primary focus is usually uterus, and hematogenically disseminated firstly to vagina then lung 1-1.5 year after the therapeutic hysterectomy in old time, and then to whole organs including liver, kidney, spleen, small intestine, skin, cardiac coronary artery, spinal cord, finally brain where the patient was fatal after longer headache than a week and sudden apnea followed by cardiac arrest, before the introduction of methotrexate (amethopterin, MTX) to treat choriocarcinoma (Ch-Ca).

\section{Methods}

\section{Chemotherapy to achieve complete remission of $\mathrm{Ch}-\mathrm{Ca}$}

The patients received $0.5 \mathrm{mg} / \mathrm{kg}$ MTX every 2 days in a week for 2-3 weeks by intramuscular injection or drip infusion, which was repeated until negative serum beta human chorionic gonadotrpine (hCG). Actinomycin D $0.5 \mathrm{mg} / \mathrm{kg}$ was combined MTX. Leucovorin $6 \mathrm{mg} / \mathrm{day}$ was injected $2 \mathrm{~h}$ after cessation of MTX injection to prevent leucopenia. High dose $15 \mathrm{mg}$ drip infusion was used every day for 10 days in the treatment of brain metastasis [1]. Combination chemotherapy that was EMA/CO (etoposide, MTX, actinomycin D, cyclophosphamide, vincristine), was used in resistant case [2]. The chemotherapy was repeated until complete remission, where the tumor disappeared in metastases and in primary focus in the uterus and serum and urinary hCG disappeared. Normal pregnancy was achieved after complete remission of primary uterine focus [1]. Primary focus and metastatic tumors were detected by chest X-ray, tumor angiography, electroencephaography (brain metastasis) etc. before real time B-mode, color Doppler imaging, 3D color Doppler ultrasound.

\section{Prophylactic MTX chemotherapy to prevent $\mathrm{Ch}-\mathrm{Ca}$}

It was performed in postmolar monitoring, 3-4 weeks after removal of hydatidiform mole. Since Ch-Ca was the most frequently developed after the complete hydatidiform mole, trophoblasts invaded in some post-molar endometrium, and $\mathrm{Ch}-\mathrm{Ca}$ was treated until complete remission, where no tumor hCG was detected, and some postmolar cases were positive in pregnancy test, the preventive MTX chemotherapy was performed. In addition postmolar patient was repeatedly examined gynecologically associating vaginal scan ultrasound and serum and urinary hCG determination as frequently as possible until 3 years after the mole, chest X-ray and ultrasound imaging of organs were studied if it is necessary, Ch-Ca preventive chemotherapy was performed. MTX was administered to reject postmolar trophoblasts, because ChCa cells disappeared after the complete remission achieved by primary chemotherapy. MTX is orally administered at least $10 \mathrm{mg}$ for 7 days to prevent Ch-Ca. Two cases, who were positive to pregnancy test, were administered high dose MTX for 200-300 mg until negative pregnancy test. Thus, there developed no $\mathrm{Ch}-\mathrm{Ca}$ in 107 postmolar MTX chemotherapy cases in 1968, while 81 control cases, who were not received MTX, developed $6 \mathrm{Ch}$-Ca cases, which was significantly more than the prophylactic chemotherapy group [3]. As randomized control

*Corresponding author: Maeda K, Department of Obstetrics and Gynecology (Emeritus), Tottori University Medical School, Yonago 3-125, Japan, Tel: 81-85922-6856; E-mail: maedak@mocha.ocn.ne.jp

Received September 19, 2017; Accepted September 21, 2017; Published September 28, 2017

Citation: Maeda K (2017) Complete Remission of Choriocarcinoma with Primary Mtx Therapy and its Prevention using Mtx. J Blood Lymph 7: 176. doi: 10.4172/2165-7831.1000176

Copyright: () 2017 Maeda K. This is an open-access article distributed under the terms of the Creative Commons Attribution License, which permits unrestricted use, distribution, and reproduction in any medium, provided the original author and source are credited. 
Citation: Maeda K (2017) Complete Remission of Choriocarcinoma with Primary Mtx Therapy and its Prevention using Mtx. J Blood Lymph 7: 176. doi: $10.4172 / 2165-7831.1000176$

Page 2 of 2

trial performed by UICC (Unio Internationalis Contra Cancerum), achieved the same results as above, the prophylaxis of $\mathrm{Ch}-\mathrm{Ca}$ is true, and it is recommendable after the hydatidiform mole.

\section{Present state of Ch-Ca development in Japan}

Since Ch-Ca was treated by MTX until complete remission, and Ch-Ca was prevented by MTX administered after hydatidiform mole, there is almost no possibility to develop Ch-Ca patient at present in Japan $[1,3]$. However, $13 \mathrm{Ch}$-Ca cases were reported among 22 districts in Japan, in the registry of Japan Society of Obstetrics \& Gynecology (JSOG) in 2013. How can we explain the facts?

\section{Discussion}

Characteristic feature was found in $\mathrm{Ch}-\mathrm{Ca}$ developing number, which was 1 or 2 cases, but not more than 2 in 8 districts in JSOG statistics. The fact will indicate that the Ch-Ca developed under some particular condition, but not due to any human error. The author noticed a fact that molar cyst was difficult to be detected by real-time ultrasound imaging in early stage of complete mole, while the molar cyst was visible by repeated ultrasound 2 weeks later (Figure 1) [46], namely, hydatidiform mole similar to blighted ovum should be repeatedly studied with B-mode ultrasound 2 weeks later to find hydatidiform mole. The color Doppler [7] and 4D live silhouette mode will be associated with real-time B-mode [8]. In another statistical report on hydatidiform mole case numbers in 1970s when ultrasound is introduced in obstetrics and gynecology, the number of moles reduced for 3 cases in 100,000 population. As 9 cases reduced in a district, of which population was suspected to be 300,000 , the reduced number of molar pregnancy in the 22 districts were $9 \times 22=198$ cases. As the $\mathrm{Ch}-\mathrm{Ca}$ developing number in the group of no MTX administration was $7 \%$ in the control of no MTX [3], the expected number of Ch-Ca was $198 \times 7 \%=13$, that matches the $\mathrm{Ch}-\mathrm{Ca}$ of 22 districts reported in 2013 by JSOG [4]. The number of Ch-Ca was reported also 13 in 2014 [5], which was the same as 2013, where there was no change between 2013 and 2014, namely, there was similar condition to produce Ch-Ca. Thus, the author's estimation of the procedure of $\mathrm{Ch}$-Ca development will be true. Therefore, a blighted ovum should be carefully studied to find hidden mole cyst, and the uterine content of blighted ovum should be histologically examined to detect hidden molar pregnancy to receive Ch-Ca preventing treatment.

\section{Conclusion}

Choriocarcinoma should be treated by MTX before hysterectomy with primary systemic chemotherapy, because the treatment is capable to achieve complete remission including the disappearance of metastases and primary uterine focus resulting normal pregnancy. Choriocarcinoma is prevented by postmolar MTX chemotherapy until the disappearance of hCG. Uterine content interrupted with the diagnosis of blighted ovum or suspected hydatidiform mole should be pathologically studied to find molar pregnancy in order to receive choriocarcinoma preventing treatment.

\section{References}

1. Maeda K, Yoshioka S, Iba N (1970) Treatment of choriocarcinoma. Jpn Obstetrics and Gynecology 20: 483-492.

2. Kondo S (1995) Saitama University, personal communication.

3. Koga K, Maeda K (1968) Prophylactic chemotherapy with amethopterin for prevention of choriocarcinoma following removal of hydatidiform mole. AJOG 100: $270-275$.

4. Japan Society Obstet Gynecol, Neoplasia Committee report. Acta Obstet Gynecol Japonica 68: 1117-1235

5. Japan Society Obstet Gynecol, Neoplasia Committee report. Acta Obste Gynecol Japonica 69: 1289-1292.

6. Tsuzaki T, Iwamoto K, Myamoto N, Takeuchi K, Oota M, et al. (1982) Studies on hydatidiform mole and blighted ovum. 1982 Congress of Japan Soc Medical Ultrasound PD-20: 839-840.

7. Maeda K, Kurjak A, Varga G (2008) Trophoblastic disease, In: Kurjak A Chervenak FA editors. Donald Textboook of Ultrasound in Obstetrics and Gynecology 2008, Jaypee, New Delhi, 244-257.

8. Yamamoto K, AboEllail MAM, Ishimura M, Tanaka T, Mori N, et al. (2017) HDlive Silhouette Inversion Mode in Diagnosis of Complete Hydatidiform Mole. Journal of Ultrasound in Medicine 36: 633-835.

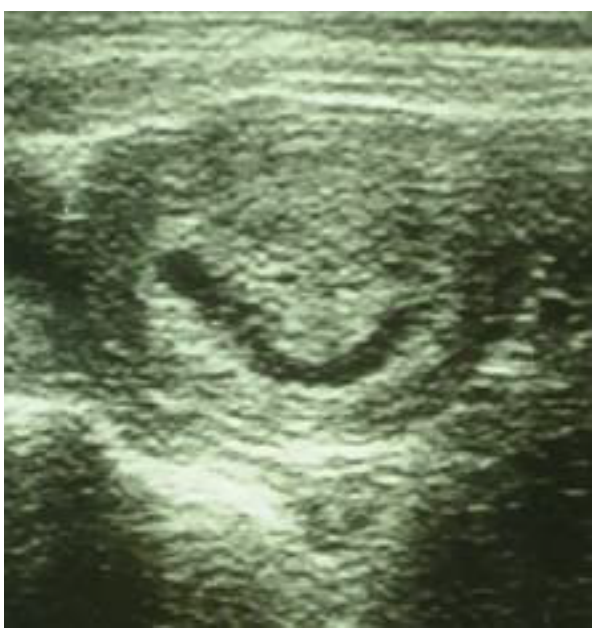

Figure 1: Ultrasound image of an complete hydatidiform mole, of which molar cyst was hardly recognized in the sonogram recorded in its early stage, where the image was similar to a blighted ovum, while the vomiting and positive pregnancy test were noted [6]. Complete molar cysts were ultrasonically detected 2 weeks later to treat it as hydatidiform mole, and enable the patient to receive choriocarcinoma preventive treatment. 